\title{
El proceso de recontextualización y la construcción de identidades pedagógicas
}

\author{
NELSONE. LOPEZ JMEÉNEZ*
}

Importante iniciar esta disertación señalando que sobre la Educación son muchos y muy variados los estudios que se han realizado. Posiblemente encontremos que difieren en cuanto al enfoque, la perspectiva, la temática, la intencionalidad, el objeto, el nivel. etc.; pero de lo que parece haber conciencia es de la reiteración y yuxtaposición de algunos de los elementos que constituyen los diferentes cuerpos de análisis.s.

El presente trabajo intenta hacel un aporte al estudio sistemático de los principios, reglas y prácticas que subyacen en el proceso de transformación de los textos y discursos del contexto primario de producción discursiva al contex10 secundario de reproducción discursiva (recontextualización) en la medida que ic asume que es en él y a través de ếl, que tienen origen las tensiones y confliclos que permiten explicar las diferentes identidades pedagógicas hoy presentes en nuestra realidad educativa colombiana. Es conveniente señalar que el análiil: se centrará en el nivel de la Educación Superior, en tanto, que el proceso de recontextualización presente en este nivel no ha sido estudiado de manera puntual.

En ese sentido se puede señalar que la estructura de esta conferencia persiIJu l. corisecución de los siguientes propósitos:

1. Presentar un manejo sistemático de los aportes de Basil Bernstein al anáIi:i: de la Educación, la Pedagogía y el Discurso Pedagógico. Estos aportes se uliucen como los aspectos básicos de la Transmisión Cultural.

Fundamentar aspectos teóricos relacionados con el proceso de incunlcxlualización y su incidencia en la Educación Superior en Colombia como wrimile central del proyecto de investigación Relaciones entre el Campo de Ari inlevilualización Oficial y el Campo de Recontextualización Pedagógica: su wis wion a la Universidad colombiana.

Nigumeritar la necesidad de avanzar en estudios sólidos sobre las Identi1.11 : ledirgógicas que se construyen en la Educación Superior en Colombia.

I' "1. Il in:azar los anteriores propósitos se considera conveniente atender el mivilo de aspectos que forman parte del horizonte teórico de esta confe11111.1 y que se constituyen en elementos centrales del estudio de las Identida-

Itwith thi litulin Universidad Surcolomblana. Actualmente adelanta estudios de Doctorado en Educain in l.u Universidald del Valle. Areahlenguaje y Educación. 
des Pedagógicas. Cada vez que hablamos de recontextualización estamos planteando la existencia de un proceso, de unas reglas. de campos específicos en los cuales ocurren los procesos de recontextualización, de la forma como esa recontextualización actúa sobre el conocimiento y de los efectos que tiene dicha recontextualización en las identidades pedagógicas o en los sujetos que son posicionados o ubicados en un determinado conocimiento, que para efectos de este análisis se denomina conocimiento oficial.

\section{El proceso de recontextualización}

Este proceso es asumido como la operación mediante la cual se mueven los textos y prácticas del contexto primario de producción discursiva, al contexto secundario de reproducción discursiva. Es un proceso que implica selección, abstracción, des-ubicación y reutsicación, des-enfoque y re-enfoque de los significados y prácticas generadas en un campo de producción. Esto significa que cuando hablamos de recontextualización de los textos, estamos queriendo decir, que éstos dejan de ser los mismos porque:

a) El texto cambia su posición en relación con otros textos, prácticas y posiciones.

b) El texto es modificado mediante reglas a las cuales ya nos hemos referido anteriormente. (Selección, simplificación. condensación y elaboración).

c) Al texto se le otorga una nueva posición y es re-enfocado.

\section{Las reglas de recontextualización}

En diferentes escenarios escolares y no escolares un asunto que reviste importancia es el de comprender cómo sucede la transformación del conocimiento -teórico, práctico, expresivo, oficial o local- en la comunicación pedagógica. El estudio de esta transformación en ocasiones le da relevancia a los contenidos que se manejan, a la transmisión de diferentes tipos de habilidades, o a las consecuencias o efectos de la transmisión pedagógica.

La teoría de la Transmisión Cultural (Bernstein y continuadores) contempla el estudio de ésta transformación desde un sistema de reglas que actúan selectivamente sobre lo que se comunica en a relación pedagógica. En el contexto de esta teoría, estas reglas son asumidas, como reglas de recontextualización $y$, dan lugar o constituyen lo que se denomina el discurso pedagógico especifico'.

El discurso pedagógico es entendido como una regla que engloba y combina dos discursos: un discurso técnico que vehicula destrezas de distintos tipos y las relaciones que las unen, y un discurso de orden social. El discurso pedagógico incluye: por una parte, reglas que crean destrezas de uno y otro tipo y reglas que regulan sus relaciones mutuas $y$, por otra, reglas que recrean un orden social.

En el contexto de esta teoría se denomina discurso instruccionalal discurso que crea destrezas especializadas y sus mutuas relaciones y discurso re- 
gulador al discurso moral que crea orden, relaciones e identidad ${ }^{3}$.

En las escuelas y en las aulas se suele hacer una distinción entre lo que llaman "transmisión de destrezas" y "transmisión de valores". Se mantienen siempre separados, como si existiese una conspiración para disimular el hecho de que sólo hay un discurso. En mi opinión, sólo hay un discurso, no dos, porque la voz secreta de este instrumento tiene que disimular el hecho de que sólo existe uno. La mayoría de los investigadores estudian continuamente los dos o creen que hay dos: como si la educación se refiriese a los valores, por una parte, y a las competencias, por otra.

(Bernstein. 1998)

Interesante afirmar que el estudio del discurso pedagógico y del proceso de recontextualización que él agencia nos permite contar con referentes teóricos básicos para el estudio de la cultura académica que subyace en la formación de nuestros profesionales y que determina la identidad de los mismos. Cómo y cuáles son los principios de organización del conocimiento: cómo se relacionan los agentes involucrados en el proceso de formación: cuáles son las prácticas pedagógicas predominantes; a qué se le dedica mayor atención La la enseñanza o al aprendizaje?4; son interrogantes que a través del estudio riguroso del discurso pedagógico se pueden absolver.

¿Cuál es la naturaleza del discurso pedagógico? El discurso pedagógico inicialmente puede ser considerado un principio que permite la reordenación de los discursos; es un principio para des-ubicar discursos, para reubicarlos y recentrarlos de acuerdo a una necesidad pedagógica concreta. Es un principio mediante el cual se apropian otros discursos y se establece entre ellos una relación, con el fin de su transmisión y adquisición selectivas. El discurso pedagógico no es física, química, biología; el discurso pedagógico no puede identificarse con lo que transmites.

El proceso de des-ubicación de un discurso, que consiste en el desplazamiento de su ubicación original a una nueva, da lugar a un cambio, a una transformación. El cambio o transformación se produce toda vez que el desplazamiento origina un vacío, un espacio, en donde entra a actuar la ideología. Ningún discurso se desplaza sin que intervenga la ideología, lo cual origina una transformación ideológica del discurso. Dicha transformación pone de manifiesto que un discurso real no mediado, se transforma en discurso mediado o imaginario.

Si se asume que la recontextualización es entendida como el proceso mediante el cual se mueven los textos y prácticas del contexto primario de producción discursiva, al contexto secundario de reproducción discursiva, es posible señalar que el discurso pedagógico es un principio recontextualizador. El discurso pedagógico está constituido por un principio recontex-

3 Un análisis pormenorizado de las relaciones entre estos discursos nos permite advertir que el discurso instruccional está incluido en el discurso regulador y que éste es el dominante. El proceso de inclusión se da, en tanto que el discurso pedagógico actúa como la regla que lleva a dicha inclusión para crear un texto, para crear un discurso.

4 Díaz y López. "La Construcción de Estructuras Curriculares Sustentadas en Investigación". Consejo Nacional de Acreditación. Cali, Abril de 1999.

5 Bernstein. Op, cit. 
tualizador que se apropia, recoloca, recentra y relaciona otros discursos para establecer su propio orden'. Esto permite reiterar que no puede identificarse nunca el discurso pedagógico con ninguno de los discursos que haya recontextualizado?.

El principio recontextualizador crea campos recontextualizadores, crea agentes con funciones recontextualizadoras $^{8}$. Estas funciones se convierten en el medio a través del cual se crea un discurso pedagógico específico. El principio recontextualizador es inherente a la constitución de los campos de recontextualización, como a los agentes involucrados en ellos con sus prácticas e ideologías.

El campo de recontextualización tiene una función crucial en tanto que actúa sobre la autonomía fundamental de la educación. Este es el problema central del desarrollo de mi proyecto doctoral' ${ }^{\natural}$ el cual persigue la realización de un estudio detenido sobre la naturaleza y las relaciones entre los campos de recontextualización oficial y pedagógica.

El Campo de Recontextualización Oficial C.R.O. es el creado y dominado por el Estado y sus agentes seleccionados de diferentes niveles. El Campo de Recontextualización P.edagógica C.R.P. es aquel compuesto por agentes pedagógicos en sus diferentes niveles incluyendo facultades, centros universitarios, departamentos de educación, revistas especializadas, fundaciones, etc.

El estudio de la relación entre estos dos campos permite la construcción de las siguientes hipótesis que serán materia de mi investigación y que aquí son trabajadas con relativo detenimiento:

- A mayor capacidad de recontextualización pedagógica mayor capacidad de autonomía.

- A menor capacidad de recontextualización pedagógica menor capacidad autonómica.

En Colombia, últimamente, se están insinuando algunas reflexiones y elaboraciones sobre los campos de recontextualización inherentes a la educación ${ }^{10}$. No obstante, es preciso advertir que estudios sólidos sobre este aspecto no se poseen y, de ahí, el interés de este trabajo por aportar algunos elementos que contribuyan a un estudio consistente del tema.

Bernstein, op, Cit.

7 Es preciso señalar que este planteamiento aporta elementos significativos al Campo Pedagógico en lo que hace referencia a la reflexión y discusión sobre la naturaleza de la pedagogía.

B El concepto de campo empleado para describir metafóricamente los contextos internos y externos de la Educación. Pierre Bourdie, lo define como un estado de relaciones de fuerza entre los agentes - las instituciones envueltas en la lucha por la distribución del capital específico que acumulado en el curso de las luchas anteriores orienta las estrategias posteriores.. Las luchas en el campo tienen por efecto el monopolio de la violencia legítima, (autoridad específica) es decir, la conservación o la subversión de la estructura de la distribución del capital especifico. Un trabajo detallado y actualizado sobre esta categoría se encuentra en el texto Respuestas por una antropología Reflexiva, Capítulo II. "La Lógica de los Campos" de Bourdie y Wacquant. Editorial Grijalbo. México, 1995.

- Relaciones entre el Campo de Recontextualización Oficial y el Campo de Recontextualización Pedagógica: su aplicación a la universidad colombiana.

10 Podrían reseñarse los estudios y documentos surgidos a raíz de la asunción de la Acreditación como un factor de calidad y excelencia. Entre otros, se pueden señalar por su aceptable continuidad, los trabajos desarrollados por ASCUN, CNA, ICFES y el conjunto de las instituciones que hacen parte 
En cuanto a la transformación que se observa de las relaciones entre el Estado y la universidad como consecuencia de los cambios surgidos en el Campo Internacional en sus diferentes sectores /económico, político, cultural, científico, tecnológico, etc.) y la repercusión que ha tenido en el ámbito nacional, es importante remitirnos a lo planteado por Myriam Henao (1999) con referencia a los posibles momentos y su caracterización". Señala la autora como momentos: los de anomia, heteronomía y autonomía y se fundamenta para dicha distinción en las normativas centrales que en el contexto del estudio realizado se toman como una política gubernamental o como una política pública.

Se busca responder a unos interrogantes que surgen en un presente transformado en lo político, en el papel del Estado. en la visibilidad de la sociedad civil y en la conformación del espacio público; que además, se complementa con un contexto académico, educativo y científico modificado tanto en sus normativas. como en sus grandes tendencias, altamente heterogéneo, segmentado y diferenciado

Como referente para analizar la transformación de la relación Estadouniversidad se pueden examinar las diferentes expresiones que ha tomado la autonomía, y cómo ésta, entendida como ejercicio de las instituciones de la educación superior, ha ido transformándose a la luz de la transformaciones de dicha relación.
La autonomía se da desde la Edad Media cuando aparece la universidad, es uno de sus principios rectores.

El que existan Estados centralizados que manipulen, encasillen, encajonen y regulen por todos los medios las formas de acción de la universidad, son la expresión de la vulneración de la autonomía o de las diferentes manifestaciones visibles o invisibles que adoptan las tensiones o relaciones de poder entre el Estado y la universidad por el dominio de la autonomía o por la regulación de la misma.

En las relaciones de poder la autonomía es como el gran premio o triunfo alrededor del cual lucha el Estado y las diferentes agencias pedagógicas. En tanto que la universidad tiene que defender su escenario se presenta una lucha por la autonomía. Qué tanto se comprime y qué tanto se expande la autonomía, depende de las relaciones de fuerza entre el Estado y la universidad.

La autonomía es un gran espacio que tiene la universidad pero en la medida que se transforman las relaciones entre Estado y universidad se puede comprimir o expandir; la autonomía está allí presente. Ésta se da en un campo de tensiones y luchas por buscar y garantizar hegemonía.

La importancia de éste análisis se evidencia significativamente en el estudio de las identidades pedagógicas que proyecta el C.R.O. y las que realmente están logrando las instituciones a través de su C.R.P. Sin duda alguna, el estudio de estas tensiones y conflictos permitirá avanzar hacia la cons- 
titución de una nueva cultura académica que permita el logro de identidades profesionales alternativas en Colombia.

En palabras de Bernstein es conveniente acotar si el C.R.P. puede producir algún efecto en el discurso pedagógico con independencia del C.R.O. habrá cierta autonomia y se producirán tensiones con respecto al discurso pedagógico y sus prácticas, pero. si sólo existe el C.R.O. no habrá autonomía. Precisamente este es el objeto básico de la investigación que me propongo adelantar.

Resulta conveniente señalar que el principio recontextualizador, además de recontextualizar el qué del discurso pedagógico también se ocupa de la recontextualización del cómo de dicho discurso. Define qué discurso debe ser pedagogizado, es decir, convertirse en materia y contenido de la práctica pedagógica y de igual manera, define la forma como debe ser la instrucción.

Esta última forma parte del discurso regulativo en tanto que encierra en sí un modelo de aprendiz, de maestro y de la relación entre estos. Es importante anotar que la selección de la teoría de la instrucción, en este sentido. no puede entenderse como algo meramente instrumental. El principio de recontextualización no sólo selecciona el qué, sino también el cómo de la teoría de la instrucción.
¿Dónde se lleva a cabo el proceso de recontextualización? Con relación a este interrogante es preciso consignar que la recontextualización se lleva a cabo dentro de un contexto específico, el cual estructura un campo o un subconjunto de campos cuyas posiciones, agencias, discursos y prácticas regulan la circulación de textos entre el contexto primario de producción y el contexto secundario de reproducción'2.

Se puede afirmar que el contexto de recontextualización se convierte en la estructura organizativa que permite la reproducción selectiva de los discursos educativos. El Estado ejerce una acción significativa en la determinación de este contexto, aunque es necesario señalar que no es el único.

Lo anterior permite agregar que la recontextualización, determinada por el Estado (oficial) no proporciona únicamente regulaciones, también proporciona límites de lo que puede entrar en el contexto de reproducción, es decir, qué puede entrar en el espacio pedagógico y qué no puede entrar.

\section{Campo de recontextualización}

Se entiende como el campo estructurado por el contexto de recontextualización, cuyas posiciones, agentes y prácticas están centrados en los movi-

12 El contexto de recontextualización conlleva a un número de campos tales como:

a) Departamentos y sub-agencias especializadas del Estado y autoridades educativas locales del Estado, con su investigación y sistema de inspectores.

b) Departamentos de educación universitaria e instituciones similares junto con su investigación.

c) Medios de educación especializados, seminarios, revistas, etc., y casas editoriales junto con sus lectores y asesores.

d) Campos no especializados en el discurso educativo pero capaces de ejercer influencia en el proceso de recontextualización. (Bernstein y Dlaz. "Hacia una teoría del discurso pedagógico". Revista Colombiana de Educación. No. 15. Bogotá, 1985). 


\section{3}

\section{Paideia Surcolombiana}

mientos de textos y prácticas del conrexto primario de producción discursiva a un contexto secundario de reproducción discursiva. La función de las diferentes posiciones, agentes y prácticas de este campo es regular la circulación de textos entre el contexto primario y el secundario ${ }^{13}$.

De una manera más detallada podemos entender el campo de Reconiextualización como el compuesto por oosiciones lopuestas y complementaras) que construyen un campo en conficto y lucha por el predominio'4.

Las diferentes posiciones que se dan en el campo de recontextualización pueden examinarse en tres niveles diferenciables a saber: autor, actor e identidad.

El autor: hace referencia al discurso autorizado. (Tecnología Educativa).

El actor: alude a los patrocinadores. (M.E.N., - /CFES).

Las identidades: son el resultado de las especializaciones pedagógicas. Operativas e Instruccionales).

Una posición en el campo constiiuye una especialización del discurso, una especialización de los actores paerocinadores y una identidad especiaizada, que adquiere su significación de as posiciones opuestas y complementarias. De lo anterior se puede deducir que los campos recontextualizadores oficiales constituyen espacios para la construcción, distribución, reproducción y cambio de las identidades pedagógicas.

Como se explicará más adelante. dichas identidades tienen una base so- cial y un curso de desarrollo. La base social está constituida por los principios del orden social y los deseos institucionalizados por el Estado en su sistema educativo. El curso de desarrollo es moral, informado y marcado por la situación. En consecuencia, la identidad pedagógica es la inclusión del curso de desarrollo en una base social.

Actualmente se puede señalar que el campo de recontextualización está constituido por una compleja tipología de modos pedagógicos con sus respectivas identidades que proyectan. Sin embargo se puede plantear la existencia de dos grandes modelos pedagógicos que permiten manejar la complejidad existente. Estos dos modelos pedagógicos son: el de actuacióny el de competencia's.

Como el propósito de ésta conferencia es analizar las diferentes identidades pedagógicas como producto del proceso de recontextualización se hace necesario pre-

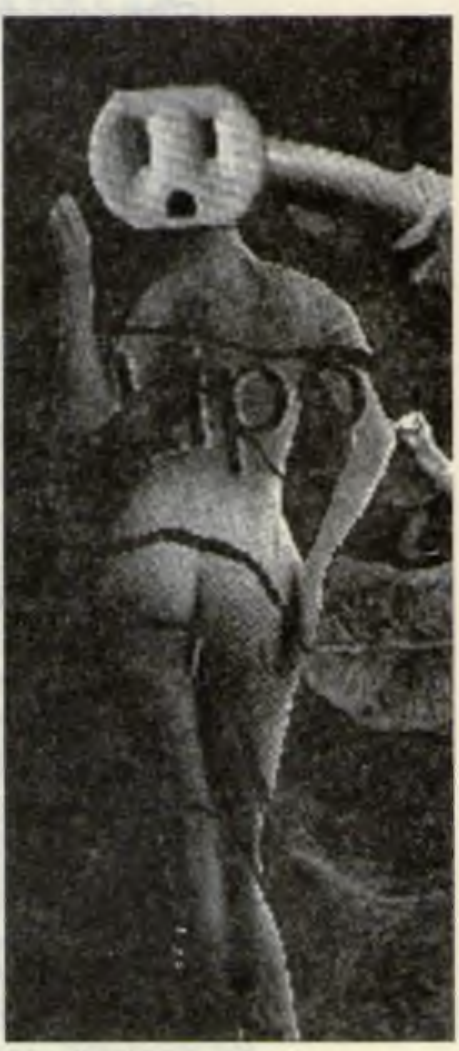
cisar la diferencia de identidad de acuerdo con el modelo pedagógico

Véase Basil Bernstein. Poder, educación y conciencia. Sociologla de la transmisión cultural. Editor. Cristian Cox. CIDE; Santiago, 1988.

Bernstein, op. Cit.

15 Un estudio interesante sobre la relación entre estos modelos se realizó en el marco de nuestro doctorado en seminarios desarrollados en la Universidad de Caldas y la Universidad Surcolombiana, que contaron con la presencia del Doctor Harry Daniels, profesor de la Universidad de Birmingan. Se espera elaborar una publicación con el material tratado en estos eventos. 
acogido. En otras palabras, entre estos dos modelos se presenta un conflicto y de la posición que se asuma frente a el, es posible determinar la identidad pedagógica resultante.

En el marco de este trabajo no se adelanta un estudio exhaustivo sobre estos modelos, sin embargo es conveniente advertir algunas características determinantes que recogen la naturaleza de los mismos.

El modelo pedagógico de actuación concede un interés fundamental al resultado específico del adquiriente a un texto concreto, que de acuerdo con las previsiones construirá el aprendiz y a destrezas especializadas necesarias para la producción de este resultado. ya sea texto o producto concreto.

El modelo pedagógico de competencia se refiere a los procedimientos para comprometerse con el mundo y construirlo. Las competencias son intrínsecamente creativas y se adquieren de forma tácita en las interacciones informales. Son logros prácticos.

El siguiente cuadro (ver página 15 ) intenta realizar una síntesis de los contrastes y diferencias entre estos dos modelos pedagógicos, que pretende dar cuenta de la dinámica del campo de recontextualización actualmente prevaleciente en la discusión sobre la construcción de identidades pedagógicas. Se toman algunas categorías que sirven de referentes diferenciadores de los modelos, como son: discurso, espacio, tiempo, evaluación, control, tex- to pedagógico, autonomía, economía, entre otros.

Importante destacar que un estudio sobre las identidades pedagógicas, necesariamente, debe dar cuenta de las posiciones que se asumen frente a las diferentes categorías utilizadas en el estudio de los modelos pedagógicos reseñados.

Lo anterior permite destacar que en el estudio de las identidades pedagógicas adquiere sentido el análisis de las diferentes tensiones que subyacen en la dinámica de la Educación Superior en Colombia ${ }^{16}$ y, que sin duda alguna, enriquecerán el horizonte de análisis de las relaciones entre el C.R.O. $y$ el C.R.P.

Resulta de particular importancia señalar que el estudio sobre el campo de recontextualización, su dinámica y su relación con el Estado, parece advertir que hoy en día, el discurso que resulte apropiado depende cada vez más de la ideología dominante en el C.R.O. y de la relativa autonomía del C.R.P.

Para el caso colombiano, la dinámica del C.R.O. parece instalar la discusión de las identidades pedagógicas en su relación con lo económico -mercado-, convirtiéndose en una de las posibles explicaciones del denominado proceso de privatización de la Educación Pública. Las siguientes cifras corroboran lo anterior:

En el período comprendido entre 1984 y 1997 la evolución de las instituciones fue la siguiente:

16 En el texto Tendencias actuales del desarrollo curricular en Colombia, publicado por el I.T.M. Medellín. febrero del 2000, se realizó un análisis de diferentes tensiones que caracterizan la dinámica de la Educación en Colombia, en la perspectiva de argumentar el carácter problemático que acompaña a todo proceso formativo. Entre otras tensiones, se analizan las siguientes:

Autonomía-heteronomía; discurso y la acción; tradición-innovación; campo de producción de conocimiento-campo de reproducción de conocimiento; discurso instruccional-discurso regulativo; pertenencia social y pertenencia académica, entre otras. 


\begin{tabular}{|c|c|c|}
\hline CATEGORIAS & $\begin{array}{l}\text { MODELO PEDAGOGICO DE } \\
\text { ACTUACIÓN }\end{array}$ & $\begin{array}{l}\text { MODELO PEDAGOGICO DE } \\
\text { COMPETENCIA }\end{array}$ \\
\hline DISCURSO & $\begin{array}{l}\text { Se manifiesta en forma de especialización de materias, } \\
\text { destrezas y procedimientos claramente marcados. Las re- } \\
\text { glas de reconocimiento y realización de textos legítimos son } \\
\text { explícitas. Su clasificación es fuerte. }\end{array}$ & $\begin{array}{l}\text { Se manifiesta en forma de proyectos, temas, ámbitos de } \\
\text { experiencia y se basa en el grupo, en el que, aparentemente, } \\
\text { los adquirientes controlan en gran medida la selección, la } \\
\text { secuenciación y el ritmo. Su clasificación es débil. }\end{array}$ \\
\hline ESPACIO & $\begin{array}{l}\text { El espacio y las prácticas pedagógicas están señalados con } \\
\text { toda claridad y regulados explícitamente. La posibilidad para } \\
\text { que los adquirientes construyan su propio espacio pedagó- } \\
\text { gico está limitada. La clasificación es fuerte. }\end{array}$ & $\begin{array}{l}\text { Hay pocos espacios definidos de modo especial. Los } \\
\text { adquirientes tienen un control considerable sobre la instau- } \\
\text { ración de espacios como lugares pedagógicos. La clasi- } \\
\text { ficación es débil. }\end{array}$ \\
\hline TIEMPO & $\begin{array}{l}\text { Está debidamente especificado. A cada actividad, deter- } \\
\text { minado tiempo. Está rígidamente definido. }\end{array}$ & $\begin{array}{l}\text { Selecciona el tiempo (gramatical) presente como modalidad } \\
\text { temporal. El tiempo no está dividido de forma explícita ni } \\
\text { estricta como marcador de las diferentes actividades; como } \\
\text { consecuencia, la división del tiempo no establece un futuro. } \\
\text { Por tanto, se subraya el tiempo presente. }\end{array}$ \\
\hline EVALUACIŌN & $\begin{array}{l}\text { La atención se otorga a lo que falta en lo que el adquiriente } \\
\text { consigue. Su clasificación es fuerte. }\end{array}$ & $\begin{array}{l}\text { El interés se centra en lo que está presente. En lo que logra } \\
\text { el adquiriente. Su clasificación es débil. }\end{array}$ \\
\hline CONTROL & $\begin{array}{l}\text { El espacio, el tiempo y el discurso dan lugar a estructuras y } \\
\text { clasificaciones explícitas y, aunque éstas pueden convertirse } \\
\text { en lugares de controversia, establecen y transmiten el orden. }\end{array}$ & $\begin{array}{l}\text { La ausencia de estructuras y clasificaciones explícitas hacen } \\
\text { que tanto la posibilidad como el empleo del control sean } \\
\text { estrategias de baja prioridad en este modelo. Esto no quiere } \\
\text { decir que no se produzcan modos de control de posición y } \\
\text { de control imperativo, sino que no constituyen modos pre- } \\
\text { ferentes. }\end{array}$ \\
\hline $\begin{array}{c}\text { TEXTO } \\
\text { PEDAGÓGICO }\end{array}$ & $\begin{array}{l}\text { Consiste en la actuación del adquiriente. Esta actuación se } \\
\text { objetiva mediante las calificaciones. Señala el pasado. }\end{array}$ & $\begin{array}{l}\text { No es tanto un producto del adquiriente cuanto que indica } \\
\text { algo diferente del mismo. }\end{array}$ \\
\hline AUTONOMIA & Es restringida. & Amplia \\
\hline ECONOMIA & $\begin{array}{l}\text { Base teórica mucho menos elaborada; por lo tanto los costos } \\
\text { son bajos. El carácter explicito de la transmisión permite que } \\
\text { estos modelos dependan menos de los atributos personales } \\
\text { del profesor, por lo que su provisión es menos restrictiva. }\end{array}$ & $\begin{array}{l}\text { Costos más altos. Se cargan al compromiso individual de los } \\
\text { profesores. Requiere una base teórica más elaborada. Los } \\
\text { aspectos comunes de procedimiento compartidos en un } \\
\text { grupo constituyen el centro de atención. }\end{array}$ \\
\hline $\begin{array}{c}\text { MODOS } \\
\text { PEDAGÓGICOS }\end{array}$ & $\begin{array}{l}\text { Difieren en el grado de especialización de sus textos. "Dife- } \\
\text { rentes a" } \\
\text { a. Singularidades } \\
\text { b. Regiones } \\
\text { c. Genérico }\end{array}$ & $\begin{array}{l}\text { Relaciones fundamentales de tipo "similar a" } \\
\text { 1. Liberal Progresista - Desarrollo- } \\
\text { 2. Populista - Reconocimiento- } \\
\text { 3. Radical - Cambio Radical de Conciencia- }\end{array}$ \\
\hline
\end{tabular}




\section{6}

Universidad Surcolombiana

Instituciones Públicas $\Rightarrow 49-85$

$=$ Incremento $173.5 \%$

Instituciones Privadas $\Rightarrow 78-187$

$=$ Incremento $240 \%$

La participación porcentual en la matrícula de la Universidad Pública en pregrado es la siguiente:

$>1965$ representaba el $56.8 \%$

$>1984$ representaba el $41.3 \%$

$>\quad 1992$ apenas el $29.6 \%^{17}$

El gráfico No. 2 intenta sistematizar el campo recontextualizador en lo relacionado con los modelos, con los modos y las identidades que éstos proyectan.

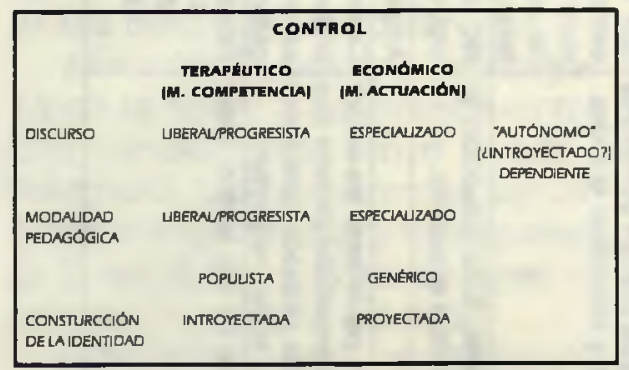

Gráfico No. 2

\section{Conocimiento oficial e Identidades pedagógicas ${ }^{18}$}

Por conocimiento oficial se entiende, el conocimiento educativo que construye el Estado y distribuye a las instituciones educativas. Las reformas académicas y curriculares han propiciado cambios en dicho conocimiento oficial, en razón que su centro de atención, sus inclinaciones y su dirección varían y, este escenario es el que permite advertir la existencia de diferentes identidades pedagógicas.

Es necesario destacar que las reformas académicas y curriculares, surgen de confrontaciones y luchas entre grupos para convertir sus intereses e inclinaciones en normas y prácticas del Estado. Por tanto se puede prever que la inclinación y el centro de atención del discurso oficial, construyan en los profesores y en los estudiantes una disposición moral, una motivación y una aspiración particulares, inmersas en sus actuaciones y prácticas, lo cual permite considerar la construcción de una(s) identidad(es) pedagógica(s). El cuadro siguiente recoge lo anteriormente planteado:

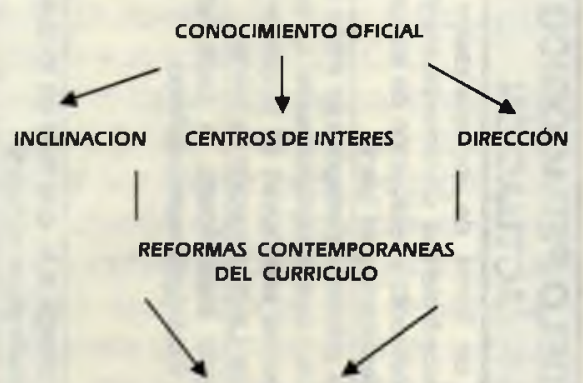

IDENTIDADES PEDAGOGICAS DIFERENTES

17 Datos tomados de los siguientes documentos:

$\checkmark$ Diagnóstico para una reforma de la Universidad Nacional. Bogorá 1985.

$\checkmark$ Estadísticas e Indicadores de la Universidad Nacional 1970-1995. Oficina de Planeación.

$\checkmark$ La financiación de la Universidad Pública. Federación Nacional de Profesores Universitarios. Septiembre de 1996.

$\checkmark$ Documento Compes de Educación Superior. Carta ICFES. No. 26 de 1995.

$\checkmark$ Un compromiso académico con la Nación colombiana. Plan global de desarrollo 1999-2003. Universidad Nacional. Julio de 1999.

18 En el texto Hacia una sociología del discurso pedagógico. Editorial Magisterio. Bogotá, abril del 2000. se retoma con detenimiento los aspectos básicos de esta reflexión que fueron ilustrados por el profesor Basil Bernstein en su conferencia pública de mayo de 1998 en el marco del Doctorado en Educación de la Universidad del Valle. Area de Lenguaje y Educación. 
Paideia Surcolombiana

La categoría identidad pedagógica es utilizada para indicar el resultado de insertar una carrera profesional en una Dase colectiva. La carrera profesional del estudiante, es una carrera de conocimientos, una carrera moral y una carrera de situación. La base colectiva de esta carrera está constituida por el principio de orden social lo en el ordenamiento de lo social, si se trata de una persuasión postmodernal que se espera o prevé transmitan las escuelas e institucionalice el Estado. La base social local de esa carrera está constituida por los ordenamientos del contexto local'19.

No resulta nuevo señalar que en las últimas décadas se han producido cambios importantes en la base social colectiva ${ }^{20}$. También se han producido cambios y transformaciones en los contextos donde se activan las carreras. ya sean contextos internacionales, nacionales, domésticos, económicos, educativos, etc. ${ }^{21}$. Lo cual nos permite advertir que el estudio de las identidades pedagógicas requiere examinar cuidadosamente dichos cambios y pre- cisar de qué manera, las diferentes reformas académicas y curriculares que se vienen agenciando consultan o no, la dinámica y el cambio de la base social y de la base local, donde se insertan o inscriben las carreras 22 .

Los gráficos siguientes pretenden aclarar lo anteriormente expuesto.

\section{IDENTIDAd PEDagógica}

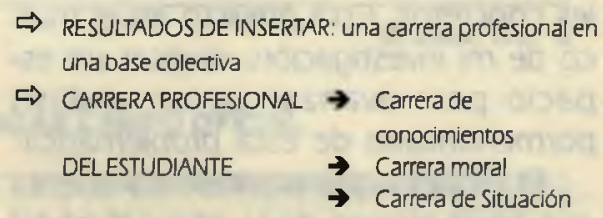

Gráflco No. 4

Una primera aproximación al estudio de las identidades pedagógicas que en Colombia se proyectan a partir del Conocimiento Oficial, puede ser adelantada apoyándonos en los planteamientos señalados por el profesor Basil Bernstein, ${ }^{23}$ en donde advierte el carácter embrionario de sus elaboraciones e invita a su complementación y enriquecimiento de las mismas a través de su aplicación a contextos loca-

13 Véase Basil Bernstein. Pedagogla, control simbólico e identidad, particularmente el capítulo sobre "Conocimiento Oficial e Identidades Pedagógicas: la politica de la recontextualización". Págs. 91-108. Editorial Morata. Madrid 1996.

¿ Véanse los estudios relacionados con los fenómenos de Globalización e Internacionalización; a manera de ejemplo se pueden consultar los siguientes: García Canclini Néstor. La Globalización Imaginada. Editorial Paidós. Colección Estado y Sociedad. Argentina, 1999; Borja Jordi y Castells Manuel. Local y Global. La gestión de las ciudades en la era de la información. Editorial TAURUS. Madrid, 1977; Garay Luis Jorge. Globalización y Crisis Lhegemonía o corresponsabilidad?. TM Editores. Bogotá, 1999; Medina Vásquez y Varela Barrios (Editores) Globalización y Gestión del Desarrollo Regional. Perspectivas Latinoamericanas. Editorial Universidad del Valie, Cali, 1977.

1. Para el caso Colombiano se puede consultar el texto Sobre la Profesión Educariva de Mario Díaz Villa, principalmente su parte introductoria. Documento preparado para el Consejo Nacional de Acreditación. Cali, Mayo de 1999.

z Esta reflexión alude perfectamente, para el caso nuestro, lo relacionado con el Decreto 272/98 en cuanto a la ldentidad Pedagógica del maestro colombiano.

« Planteamientos desarrollados en la conferencia pública dictada en el marco del Doctorado en Educación. Area Lenguaje y Educación, en mayo de 1998 en la ciudad de Cali. Colombia. 


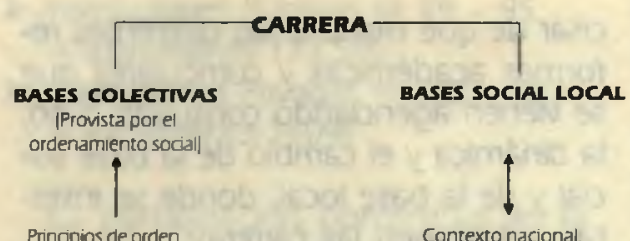

Gráflco No. 5

les concretos. Está previsto en el marco de mi investigación, dedicar un espacio para avanzar en un análisis pormenorizado de esta problemática.

El modelo que plantea, da cuenta que en el terreno de lo oficial (Estado) se pueden considerar inicialmente cuatro tipos de identidades pedagógicas que difieren entre sí, en tanto, que son resultado de una mirada particular y diferenciada de los elementos estructurantes de las mismas.

Dicho de otra manera, no asumen un tratamiento similar en el manejo de los aspectos que las pueden configurar, como son los recursos, el tiempo, la finalidad del proceso de recontextualización, la base social, su inclinación y centro de atención, su dirección. entre otros.

Los gráficos 6 y 7 aspiran a sintetizar los elementos constituyentes del modelo planteado por el profesor Bernstein.

Importante advertir que el modelo da cuenta también de las identidades pedagógicas que se construyen "externas" o por fuera del campo oficial. Dados los alcances de esta conferencia no son tratadas, lo cual no puede entenderse como un asunto que no sea importante y aportante al estudio de la construcción de las identidades pedagógicas, más aún, sabiendo que en Colombia, hoy por hoy, la educación Superior está en manos de la iniciativa privada. La decisión se toma teniendo en cuenta los referentes metodológicos suministrados.

Las directivas del Doctorado en lo que hace referencia al tiempo asignado a esta sustentación.

No obstante, sin duda alguna, en el desarrollo de la investigación será de obligado tratamiento el estudio de esta realidad académica y social.

\section{A manera de concluslón}

Hasta aquí se ha intentado exponer un referente teórico y conceptual que permita contribuir a la construcción de una gramática que de cuenta de las tensiones y conflictos que se presentan entre el C.R.O. y el C.R.P.

Se advierte, en el desarrollo precedente, la importancia de entender el proceso de recontextualización como el resultado de unas reglas derivadas de la naturaleza del poder y del control, que se erigen como procesos determinantes en la transmisión, reproducción y transformación cultural y que dan cuenta del papel que desempeña la escuela como agente y factor del mismo.

Al entender los alcances y objetivos de esta conferencia, ingenuo sería pensar que se ha agotado la temática analizada, por el contrario, se es consciente que el estudio apenas se inicia, como también, la perseverancia y dedicación indiscutibles que su trabajo serio y responsable exige. 


\section{9}

Paideia Surcolombiana

\section{CLASIFICACIÓN}

Restringida

Retrospectiva

Conervadora antigual

\section{Estado re-centrado}

Seleccionada

Prospectiva

(Neoconservadora)

Integrada

Descentrada (terapéutica)

(Profesionales)

Gráfico No. 6

\section{IDENTIDADES PEDAGOGICAS CAMPO OFICIAL}

\begin{tabular}{|c|c|c|c|c|c|c|}
\hline ITायक & RECURSOS & TIEKTO & RUC & CARACTERISTICAS & BASE SOCIAL & $\begin{array}{l}\text { INCLINACION } \\
\text { CT., AT., } \\
\text { DIREC. }\end{array}$ \\
\hline E21ROSPECTNA & $\begin{array}{l}\text { CENTRADOS } \\
\text { Orandes } \\
\text { narraciones } \\
\text { nacionales, } \\
\text { religiosas y } \\
\text { culturales }\end{array}$ & PASADO & $\begin{array}{c}\text { Estabilizar el } \\
\text { pasado en el } \\
\text { presente }\end{array}$ & $\begin{array}{l}\text { El discurso no entra } \\
\text { en relación can la } \\
\text { economía }\end{array}$ & COLECTIVA & $\begin{array}{l}\text { Control rigido } \\
\text { sobre las } \\
\text { entradas de la } \\
\text { educación. } \\
\text { * Contenidos. } \\
\text { " Prácticas } \\
\text { jerárquicamente } \\
\text { ordenadas. }\end{array}$ \\
\hline WOSPECTNA & CENTRADOS & $\begin{array}{l}\text { PASADO } \\
\text { (Diferente) }\end{array}$ & $\begin{array}{c}\text { Selectiva de } \\
\text { características de } \\
\text { pasado }\end{array}$ & $\begin{array}{l}\text { Defender y elevar el } \\
\text { rendimiento } \\
\text { económico }\end{array}$ & $\begin{array}{l}\text { NUEVA BASE } \\
\text { SOCIAL } \\
\text { COLECTIVA } \\
\text { CARRERAS } \\
\text { Sumergidas en } \\
\text { un pasado } \\
\text { Especialmente } \\
\text { selaccionado }\end{array}$ & $\begin{array}{l}\text { * Afrontar el } \\
\text { cambio cultural, } \\
\text { económico y } \\
\text { tecnológlco, } \\
\text { * Particlpación } \\
\text { en el cambio } \\
\text { contemporáneo }\end{array}$ \\
\hline $\begin{array}{l}\text { ERCADO. } \\
\text { 2ESENTRADAS }\end{array}$ & $\begin{array}{l}\text { DESCENTRADOS } \\
\text { Crean } \\
\text { estratificaciones }\end{array}$ & PRESENTE & $\begin{array}{l}\text { La práctica } \\
\text { pedagógica } \\
\text { dependerá del } \\
\text { mercado en el } \\
\text { que se adquiere } \\
\text { la identidad }\end{array}$ & $\begin{array}{l}\text { * Sistema de gestión } \\
\text { Jerárquico. } \\
\text { * Gestión recompensa } \\
\text { y castigos. } \\
\text { * Crean una nueva } \\
\text { estratificac ión tanto } \\
\text { del saber como de las } \\
\text { Identidades. }\end{array}$ & $\begin{array}{l}\text { VALOR DE } \\
\text { CAMEIO ENEL } \\
\text { MERCADO }\end{array}$ & $\begin{array}{l}\text { * Imouts que } \\
\text { optimicen el } \\
\text { yalor de cambio } \\
\text { en el mercado. } \\
\text { " Cultura y } \\
\text { contextos } \\
\text { orientados a } \\
\text { facllitar la } \\
\text { supenvivencia } \\
\text { del más } \\
\text { adaptado de } \\
\text { acuerdo con las } \\
\text { dernandas dol } \\
\text { mercado. } \\
\text { *Costo plazo, } \\
\text { extrínseco. } \\
\text { "Exploración } \\
\text { de las } \\
\text { aplicaclones } \\
\text { profesionales y } \\
\text { no de la } \\
\text { exploración del } \\
\text { conocimiento. } \\
\text { * Contrato, no } \\
\text { compromiso. }\end{array}$ \\
\hline $\begin{array}{l}\text { IERAPEUTICAS } \\
\text { DESCENTRADAS }\end{array}$ & DESCENTRADOS & PRESENTE & $\begin{array}{l}\text { - Va en contra de } \\
\text { las categorias } \\
\text { especializadas de } \\
\text { dlscurso y de } \\
\text { estratificación de } \\
\text { grupos } \\
\text { * Proyecta unas } \\
\text { identidades } \\
\text { estables } 8 \\
\text { integradas con } \\
\text { prácticas } \\
\text { cooperativas } \\
\text { adaptables }\end{array}$ & $\begin{array}{l}\text { La transmisión prefiere } \\
\text { unos límites débiles, la } \\
\text { integración prefiere } \\
\text { referirse a regiones de } \\
\text { conocimiento, a áreas } \\
\text { de experiencia. }\end{array}$ & $\begin{array}{c}\text { Cooperativa } \\
\text { Integrada }\end{array}$ & $\begin{array}{l}\text { = Estilo de } \\
\text { gestión blando } \\
\text { * Jerarquías } \\
\text { borrosas. } \\
\text { * Redes do } \\
\text { comunicación y } \\
\text { relaciones } \\
\text { interpersonales } \\
\text { disimulan el } \\
\text { poder. }\end{array}$ \\
\hline
\end{tabular}

